\title{
Edge Functionalization of Structurally Defined Graphene Nanoribbons for Modulating the Self-Assembled Structures
}

DOI:

10.1021/jacs.7b09031

\section{Document Version}

Accepted author manuscript

Link to publication record in Manchester Research Explorer

Citation for published version (APA):

Keerthi, A., Boya, R., Rizzo, D., Diez Cabanes, V., Hou, I. C-Y., Beljonne, D., Cornil, J., Casiraghi, C., Baumgarten, M., Müllen, K., \& Narita, A. (2017). Edge Functionalization of Structurally Defined Graphene Nanoribbons for Modulating the Self-Assembled Structures. Journal of the American Chemical Society. https://doi.org/10.1021/jacs.7b09031

\section{Published in:}

Journal of the American Chemical Society

\section{Citing this paper}

Please note that where the full-text provided on Manchester Research Explorer is the Author Accepted Manuscript or Proof version this may differ from the final Published version. If citing, it is advised that you check and use the publisher's definitive version.

\section{General rights}

Copyright and moral rights for the publications made accessible in the Research Explorer are retained by the authors and/or other copyright owners and it is a condition of accessing publications that users recognise and abide by the legal requirements associated with these rights.

\section{Takedown policy}

If you believe that this document breaches copyright please refer to the University of Manchester's Takedown Procedures [http://man.ac.uk/04Y6Bo] or contact uml.scholarlycommunications@manchester.ac.uk providing relevant details, so we can investigate your claim.

\section{OPEN ACCESS}




\title{
Edge Functionalization of Structurally Defined Graphene Nanorib- bons for Modulating the Self-Assembled Structures
}

\author{
Ashok Keerthi, ${ }^{\dagger, \S, \downarrow}$ Boya Radha, ${ }^{\S}$, Daniele Rizzo, ${ }^{*}$ Hao Lu, ${ }^{\dagger}$ Valentin Dies Cabanes,, Ian Cheng-Yi \\ Hou ${ }^{\dagger}$ Jérôme Cornil," David Beljonne, ${ }^{\prime}$ Cinzia Casiraghi, ${ }^{\#}$ Martin Baumgarten, ${ }^{\dagger}$ Klaus Müllen, ${ }^{\dagger}, *$ \\ Akimitsu Narita ${ }^{\dagger}, *$

\begin{abstract}
${ }^{\dagger}$ Max Planck Institute for Polymer Research, Ackermannweg 10, 55128 Mainz, Germany.
${ }^{\S}$ National Graphene Institute, University of Manchester, Booth Street East, Manchester M13 9PL, United Kingdom.

${ }^{\ddagger}$ School of Physics and Astronomy, University of Manchester, Oxford Road, Manchester M13 9PL, United Kingdom.

${ }^{\#}$ School of Chemistry, University of Manchester, Oxford Road, Manchester M13 9PL, United Kingdom.

"Laboratory for Chemistry of Novel Materials, University of Mons, Place du Parc 20, B-7000 Mons, Belgium.
\end{abstract}

Supporting Information Placeholder

\begin{abstract}
Edge functionalization of bottom-up synthesized graphene nanoribbons (GNRs) with antraquinone (AQ) and naphthalene/perylene monoimide (NMI/PMI) units has been achieved through a Suzuki coupling of polyphenylene precursors bearing bromo groups, prior to the intramolecular oxidative cyclodehydrogenation. High efficiency of the substitution has been validated by MALDI-TOF MS analysis of the functionalized precursors and FT-IR, Raman and XPS analyses of the resulting GNRs. Moreover, AFM measurements demonstrated the modulation of the selfassembling behavior of the edge-functionalized GNRs, revealing that GNR-PMI formed an intriguing rectangular network. This result suggests the possibility of programming the supramolecular architecture of GNRs by tuning the functional units.
\end{abstract}

Bottom-up molecular synthesis of graphene nanoribbons (GNRs), namely nanometer-wide graphene strips, has been attracting a growing attention for its high capability of fabricating atomically precise GNR structures with promising electronic, optical, and magnetic properties. ${ }^{1-10}$ In particular, GNRs have non-zero bandgap, which can be tuned by changing their structures, in stark contrast to zero-bandgap graphene. ${ }^{1,11}$ While the top-down methods such as "cutting" of graphene ${ }^{12}$ and "unzipping" of carbon nanotubes ${ }^{13}$ can only provide rather undefined and randomly shaped GNRs, uniform and highly defined GNRs can be obtained through the oxidative cyclodehydrogenation of tailor-made polyarylene precursors, either in solution by the Scholl reaction ${ }^{4,5,14,15}$ or thermally on metal surfaces. ${ }^{2,3,7-9}$ In particular, a solution synthesis based on the $A B$-type Diels-Alder polymerization has enabled the preparation of exceptionally long (up to >600 nm) GNRs with tunable width (ca. 1-2 nm) and optical bandgaps (ca. 1.2-1.9 eV). ${ }^{14-18}$ Such GNRs are dispersible in organic solvents, and thus can be characterized in and processed from a liquid phase.

Peripheral functionalization of organic compounds is tremendously important in the field of organic materials for largely altering as well as fine-tuning their electronic, optical, and mechanical properties for various applications. ${ }^{19}$ Moreover, appropriate pendant groups incorporated in supramolecular networks or polymers can pre-program their assembly into desired architectures. ${ }^{20}$ Considering that the bottom-up GNRs are a kind of organic polymers, substitution of their edge with different groups would also allow further modulation of their properties, including the supramolecular organization. However, there are thus far only a few examples for such edge functionalization of GNRs, except for the conven- tional alkyl substitution, namely peripheral chlorination ${ }^{21}$ and introduction of carboxylic acid or nitronyl nitroxide (NN) radicals. $^{22-24}$

Scheme 1. Synthetic protocol for the edge-functionalization of GNRs with AQ, NMI, and PMI units

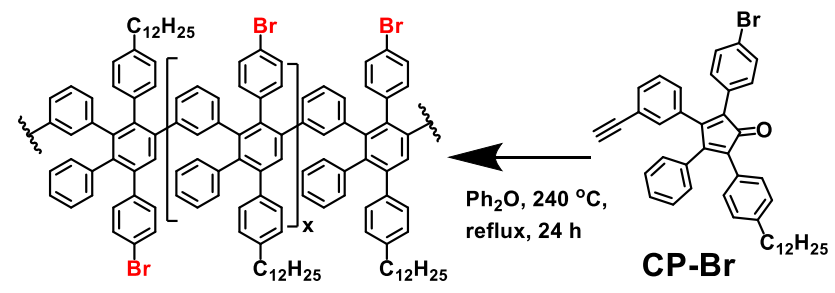

PP-Br
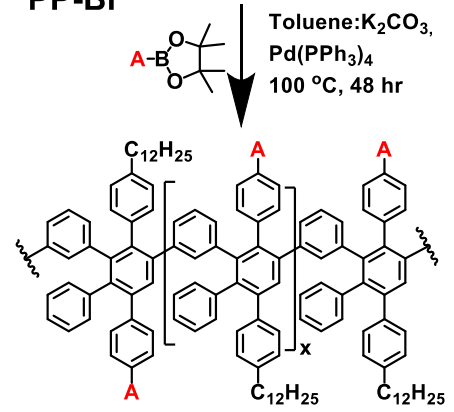

PP-A
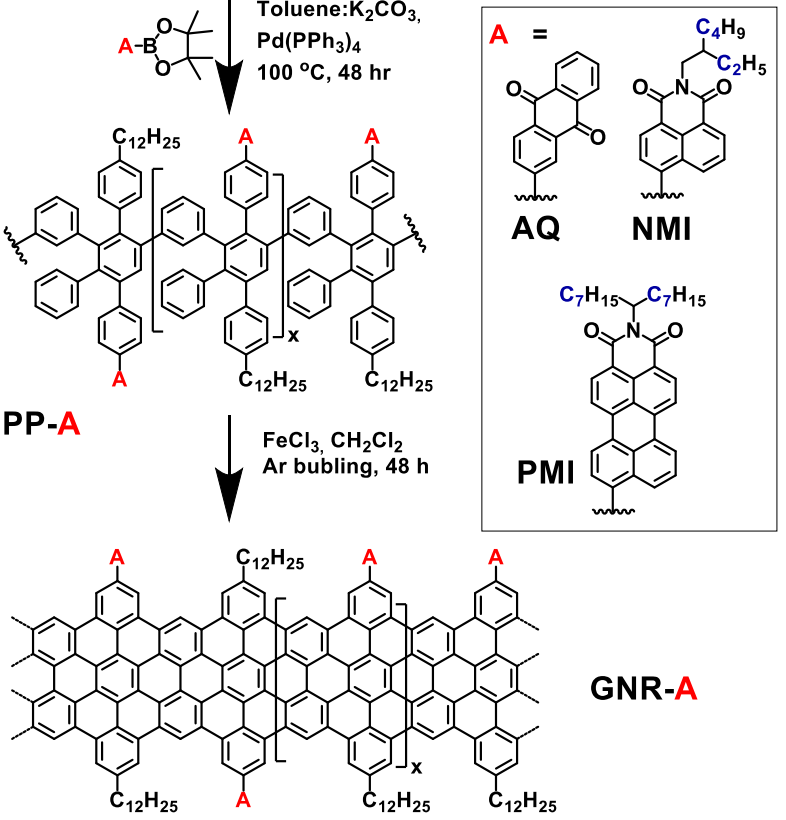

Here we report a versatile protocol to functionalize the GNR edges with different group, through Suzuki coupling prior to the cyclodehydrogenation. We demonstrate successful introduction of anthraquinone (AQ), naphthalene monoimide (NMI), and perylene monoimide (PMI) units at the peripheral positions, which reveals an unprecedented effect on the self-assembly behavior of the GNRs. 
For the synthesis of GNRs appended with NN radicals, we have recently developed a polyphenylene precursor bearing one bromo group and one dodecyl chain per repeating unit (PP-Br), through the $A B$-type Diels-Alder polymerization of tetraphenylcyclopentadienone-based monomer CP-Br (Scheme 1). ${ }^{24}$ Nevertheless, the method used for introducing the NN radical, mediated by an $\mathrm{Au}$ complex, is not immediately applicable to other functional groups, which motivated us to consider a use of more versatile Suzuki coupling. To this end, we have previously carried out a model study on the peripheral functionalization of hexa-perihexabenzocoronene (HBC) through the Suzuki coupling both before and after the cyclodehydrogenation, with boronic esters of AQ, NMI, and PMI. ${ }^{25}$ Such electron-deficient groups were selected in the view of making less-explored n-type GNR materials, which was supported by the calculations with the hybrid density functional theory (DFT). For example, substitution with the NMI or PMI unit was predicted to lower the energy levels of the GNR by $0.4-0.5 \mathrm{eV}$ (see SI for the theoretical results).

Based on the successful results with the HBC, PP-Br was thus subjected to the Suzuki coupling with three boronic esters 2(4,4,5,5-tetramethyl-1,3,2-dioxaborolan-2-yl)anthracene-9,10-

dione (AQ-BPin), N-(2-ethylhexyl)-4-(4,4,5,5-tetramethyl-1,3,2dioxaborolan-2-yl)naphthalene-1,8-dicarboximide (NMI-BPin), and $\mathrm{N}$-(1-heptyloctyl)-9-(4,4,5,5-tetramethyl-1,3,2-dioxaborolan2-yl)perylene-3,4-dicarboximide (PMI-BPin), using tetrakis(triphenylphosphine)palladium as catalyst and 2dicyclohexylphosphino-2',6'-dimethoxybiphenyl (SPhos) as ligand, in a mixture of toluene, ethanol, and $1 \mathrm{M}$ aqueous solution of $\mathrm{K}_{2} \mathrm{CO}_{3}$ (Scheme 1). After extensive purification and removal of small-molecular-weight fractions by reprecipitation, and also with recycling preparative size exclusion chromatography (SEC) when possible (see SI), functionalized polyphenylenes PP-A $(\mathbf{A}=\mathbf{A Q}$, NMI, and PMI) were obtained (Table S1).

Matrix-assisted laser desorption/ionization time-of-flight (MALDI-TOF) mass spectrometry (MS) analysis (in reflectronmode) of a low-molecular-weight fraction of PP-AQ has demonstrated a series of peaks from oligomers with up to 14 repeating units (Figure 1). The $\mathrm{m} / \mathrm{z}$ and intervals were consistent with the expected values. The isotopic distributions were also generally in agreement with the simulated patterns, as displayed for the pentamers in the insets of Figure 1, validating the successful functionalization of the polyphenylene precursors with the AQ units. Notably, the MS spectrum showed no peak corresponding to PP$\mathbf{B r}$ or partially substituted or debrominated oligomers, marking the high efficiency of the functionalization. Although the MALDI-TOF MS analysis of PP-NMI and -PMI was more difficult presumably due to stronger aggregation, expected patterns of mass could be observed for an oligomeric mixture of PP-NMI in the linear-mode (Figure S4). NMR of the three PP-A could not be properly measured even at $120^{\circ} \mathrm{C}$ in $\mathrm{C}_{2} \mathrm{D}_{2} \mathrm{Cl}_{4}$, probably also due to the aggregation, but the MS results combined with the observation of characteristic IR peaks (Figures S6-S8) indicated successful introduction of the functional groups.

The three PP-A were subsequently "graphitized" into GNR-A (A = AQ, NMI, and PMI), through the oxidative cyclodehydrogenation using $\mathrm{FeCl}_{3}$ in dichloromethane and nitromethane, following the literature protocol. ${ }^{14,25}$ It must be noted that $\mathbf{P P}-\mathbf{B r}$, and thus also PP-A were obtained as mixtures of regioisomers because of two possible orientations of the unsymmetrical $\mathbf{C P}-\mathbf{B r}$ on each Diels-Alder cycloaddition step. However, all the regioisomers of PP-A could lead to GNR-A with the uniform GNR core structure $^{14}$, although having a statistical distribution for the orientation of the dodecyl and the other pendant groups (Scheme S1). According to the previous model study with HBC, the AQ, NMI, and PMI units used here are chemically stable under the oxidative cyclodehydrogenation condition with $\mathrm{FeCl}_{3}$ and form no extra C$\mathrm{C}$ bond with the aromatic core, which would potentially make a five-membered ring. ${ }^{25}$ To explore the possibility of the postfunctionalization of the GNRs, we also attempted a synthesis of
GNR-PMI through the prior cyclodehydrogenation of PP-Br to GNR-Br (Figure S1), followed by the Suzuki coupling with PMIBPin (named as GNR-PMI*, Scheme S4). All the three functionalized GNRs, GNR-AQ, -NMI and -PMI could be dispersed in organic solvents such as tetrahydrofuran (THF), chlorobenzene, 1,2,4-trichlorobenzene (TCB), and 1,4-dioxane (see SI).

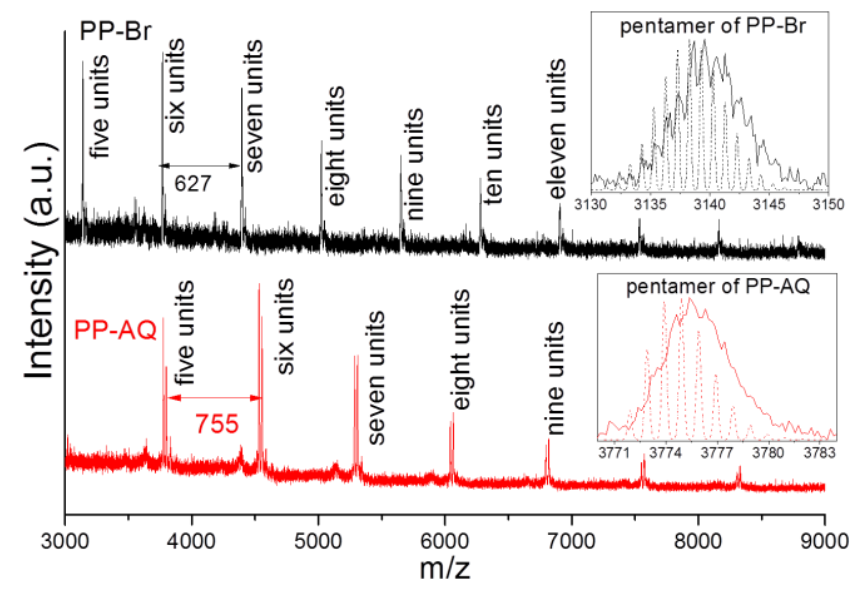

Figure 1. MALDI-TOF spectra of PP-Br and PP-AQ. Peaks with added sodium ion are also visible. ${ }^{14}$ Inset: calculated (dashed line) and observed (solid line) isotopic distribution of the pentamers.

These GNRs were initially characterized by Fourier transform infrared (FT-IR) and Raman spectroscopy (Figure 2c and S5S10). FT-IR analysis revealed the successful cyclodehydrogenation of the polyphenylene backbones to GNR core structures, displaying a clear disappearance/attenuation of out-of-plane (opla) $\mathrm{C}-\mathrm{H}$ deformation bands at around 698 and $795 \mathrm{~cm}^{-1}$ (Figures S5-S8). ${ }^{14}$ Evidences for the functionalization of the GNRs could be obtained by the observation of $\mathrm{C}=\mathrm{O}$ stretching bands, which is absent in the GNR core, at $1672 \mathrm{~cm}^{-1}$ for GNR-AQ, at 1660 and $1703 \mathrm{~cm}^{-1}$ for GNR-NMI, and at 1653 and $1691 \mathrm{~cm}^{-1}$ for GNR-PMI (Figure S5). ${ }^{26}$ On the other hand, GNR-PMI* displayed a spectrum overall resembling that of GNR-Br, rather than that of GNR-PMI, with additional bands of PMI units (Figure S9). This result most probably indicates that the postfunctionalization after the cyclodehydrogenation is not as efficient as the pre-functionalization.

Raman spectra of GNR-AQ, -NMI, and -PMI show the firstorder D and $\mathrm{G}$ as well as the second-order 2D, D + D', and 2D' peaks, which are generally in agreement with the spectra of GNR$\mathbf{B r}$ and previous solution-synthesized GNRs, ${ }^{14,15,27}$ without significant changes upon the functionalization with different groups (Figure S10). In contrast, characteristic peaks in the lowenergy regions, as represented by the Radial-Like Breathing Mode (RLBM) dependent on the GNR width, ${ }^{28,29}$ change significantly (Figure 2c). GNR-Br displayed a prominent RLBM peak at about $240 \mathrm{~cm}^{-1}$, in close agreement with that of the same GNR substituted only with dodecyl chains (GNR-C12) observed at about 230 $\mathrm{cm}^{-1} .{ }^{27}$ A RLBM peak at $\sim 236 \mathrm{~cm}^{-1}$ was still visible for GNR$\mathbf{A Q}$, but no clear peak could be unambiguously assigned to the RLBM for GNR-PMI and -NMI, which could be related to the size of the pendant units: Relatively large functional groups, such as PMI may directly slow or quench, the RLBM, ${ }^{27}$ or deactivate it through structural deformation of the GNR core. Additionally, a prominent RLBM peak was observed for GNR-PMI*, like that of GNR-Br (Figure S11). This result suggests the low efficiency of the post-functionalization in agreement with the FT-IR results, and simultaneously verifies that the RLBM peak was "quenched" by the covalently bonded PMI units.

Further evidences of functionalization could be obtained by X-ray photoelectron spectroscopy (XPS) analysis (Figure 2a and b). C $1 \mathrm{~s}$ high resolution spectra displayed slight shift of the $\mathrm{sp}^{2}$ binding energy from GNR-Br $(284.5 \mathrm{eV})$ to GNR-NMI $(284.3 \mathrm{eV})$, and then to GNR-PMI (284.1 eV), which is most probably the effect 
of the varying functional groups attached to the aromatic GNR core. Moreover, $\mathrm{C} 1 \mathrm{~s}$ peaks assignable to the imide carbon (marked with light green in Scheme 1) could be observed together with N 1s peaks for GNR-NMI and -PMI, and not for GNR-Br and $\mathbf{- A Q}$. Based on the N/C ratios, the degree of functionalization could be estimated for GNR-NMI and -PMI to be 93 and $92 \%$, respectively (see SI for more details).
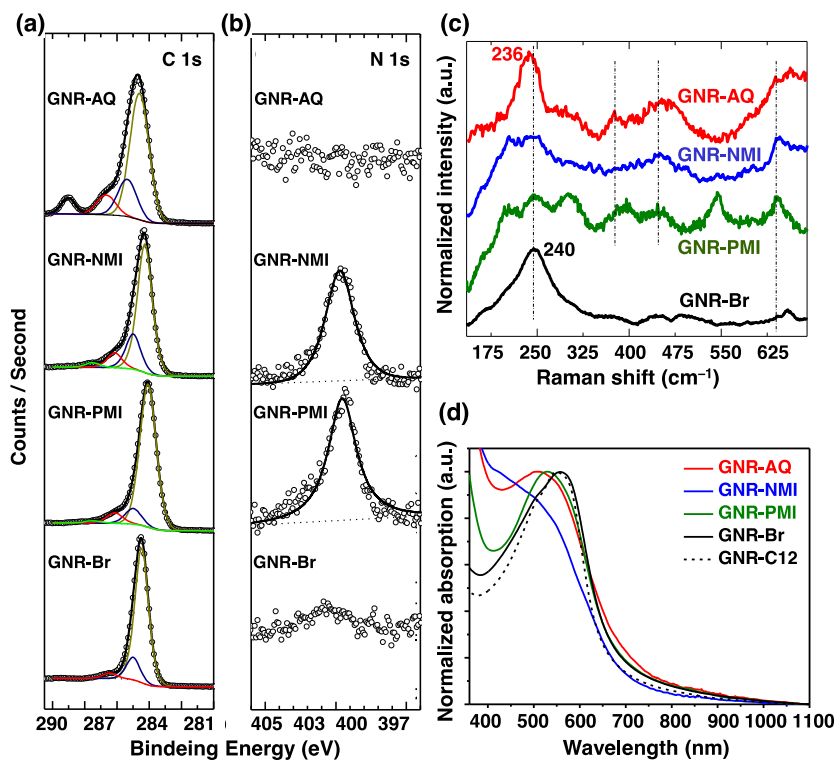

Figure 2. (a) C 1s and (b) N 1s XPS (circle: raw data; black: fitted curve; for C 1s spectra: dark yellow: $\mathrm{sp}^{2}$; navy: $\mathrm{sp}^{3}$; red: $\mathrm{sp}^{2}$ at $\mathrm{C}-$ $\mathrm{Br}$ for GNR-Br; see the color code in Scheme 1 for other GNRs), (c) magnified Raman spectra in the low-energy region (at 2.33 $\mathrm{eV}$ ), and (d) normalized UV-vis-NIR absorption spectra in NMP of the functionalized GNRs.

UV-vis-near infrared (NIR) absorption spectra of GNR-AQ and PMI in NMP dispersions displayed absorption maxima at 506 and $530 \mathrm{~nm}$, respectively, with shoulder peaks corresponding to the absorption maxima of GNR-Br and -C12 (Figure 2d). GNRPMI* also had a blue-shifted absorption maximum like that of GNR-PMI, which indicated that this peak is not derived from partially fused GNRs, but from the PMI units (Figure S14). Furthermore, the lack of shift in the absorption spectra agreed with the theoretical calculations (see SI for the details). On the other hand, GNR-NMI exhibited rather broadened absorption with a shoulder peak and the absorption edge consistent with those of GNR-C12. The spectrum at around 400-500 nm might originates from red-shifted absorption of the NMI units due to aggregation and/or interaction with the GNR core, although the possibility of "defects", namely unclosed C-C bonds, cannot be excluded.

In order to study the self-assembly behavior of the functionalized GNRs, their dispersions in TCB were drop-cast over freshly peeled graphite surfaces. AFM imaging revealed that GNR-AQ and -NMI can form closely packed assembly (Figures 3a,c and S19) similar to our previous observations with GNR-C12. ${ }^{14}$ In contrast, the deposition of the GNR-PMI in the same way reproducibly provided self-assembled rectangular networks, where single strands or bundles of GNRs crossed perpendicularly to each other (Figure 3e, S19, and S20). The widths observed for the ribbons are GNR-PMI $(6.1 \mathrm{~nm})>$ GNR-NMI $(5.1 \mathrm{~nm})$, in agreement with the simulated values (Figures S21 and S22). GNR-PMI showed the length of up to $1 \mu \mathrm{m}$, although it could be due to an end-to-end overlap of individual GNRs or alignment of a few GNRs to form such supramolecular wires. The intriguing rectangular assembly of GNR-PMI might be due to the additional $\pi-\pi$ interactions between the GNR cores and the PMI units, which are perpendicularly connected to the GNRs (Figure S23). The absence of such assembly with the NMI units indicates that ex- tending the aromatic core of the pendant units can modulate the supramolecular architecture of the GNRs in such a drastic manner. Furthermore, isolated individual strand of GNR-PMI could be facilely found under AFM analysis, which is an important step forward toward the application of the GNRs in the future nanoelectronics.
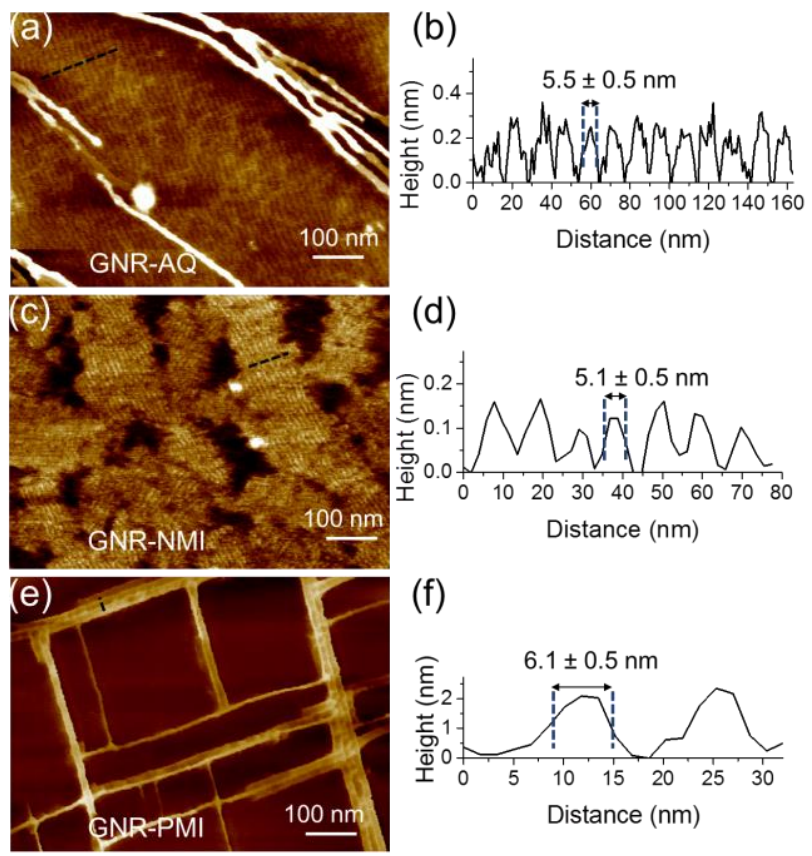

(d)

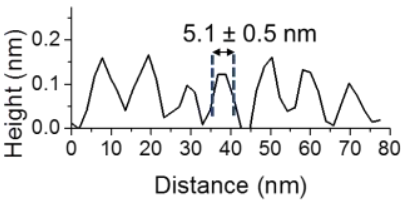

(f)

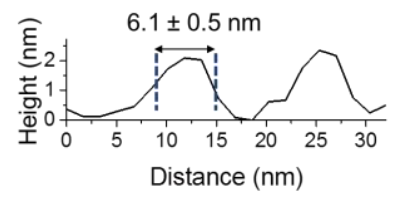

Figure 3. AFM height image of functionalized GNRs drop-casted on graphite in TCB solvent and annealed at $100{ }^{\circ} \mathrm{C}$ demonstrates highly organized self-assembled monolayers of straight and uniform nanoribbons.

In summary, we have demonstrated successful edge functionalization of GNRs, based on the Suzuki coupling prior to the cyclodehydrogenation, which could be evidenced by a combination of MS and different spectroscopic characterizations. Notably, GNRPMI revealed unprecedented self-assembly behavior, leading to rectangular network of GNRs. These results indicate the possibility of arbitrarily programming the self-assembled architectures of GNRs by the edge functionalization with proper pendent groups.

\section{ASSOCIATED CONTENT}

\section{Supporting Information}

The Supporting Information is available free of charge on the ACS Publications website.

\section{AUTHOR INFORMATION}

\section{Corresponding Author \\ *muellen@mpip-mainz.mpg.de \\ *narita@mpip-mainz.mpg.de}

\section{Notes}

The authors declare no competing financial interests.

\section{ACKNOWLEDGMENT}

We are grateful for the financial support from the Max Planck Society, the ERC grant on NANOGRAPH, EC through the Graphene Flagship, iSwitch (GA No. 642196), and MoQuaS (FP7 FET-ICT-2013-10 610449), and the Office of Naval Research BRC Program. AK would like to thank Xinliang Feng (TU Dresden) for discussion and support. RB is thankful to Leverhulme trust for early career fellowship.

\section{REFERENCES}


(1) Narita, A.; Wang, X.-Y.; Feng, X.; Mullen, K. Chem. Soc. Rev. 2015, 44, 6616.

(2) Ruffieux, P.; Wang, S.; Yang, B.; SánchezSánchez, C.; Liu, J.; Dienel, T.; Talirz, L.; Shinde, P.; Pignedoli, C. A.; Passerone, D.; Dumslaff, T.; Feng, X.; Müllen, K.; Fasel, R. Nature 2016, 531, 489.

(3) Chen, Y.-C.; Cao, T.; Chen, C.; Pedramrazi, Z.; Haberer, D.; de OteyzaDimas, G.; Fischer, F. R.; Louie, S. G.; Crommie, M. F. Nat Nano 2015, 10, 156.

(4) Vo, T. H.; Shekhirev, M.; Kunkel, D. A.; Morton, M. D.; Berglund, E.; Kong, L.; Wilson, P. M.; Dowben, P. A.; Enders, A.; Sinitskii, A. Nat. Commun. 2014, 5, 3189.

(5) Gao, J.; Uribe-Romo, F. J.; Saathoff, J. D.; Arslan, H.; Crick, C. R.; Hein, S. J.; Itin, B.; Clancy, P.; Dichtel, W. R.; Loo, Y.-L. ACS Nano 2016, 10, 4847.

(6) Jordan, Robert S.; Wang, Y.; McCurdy, Ryan D.; Yeung, Michael T.; Marsh, Kristofer L.; Khan, Saeed I.; Kaner, Richard B.; Rubin, Y. Chem 2016, 1, 78.

(7) de Oteyza, D. G.; Garcia-Lekue, A.; Vilas-Varela, M.; Merino-Diez, N.; Carbonell-Sanroma, E.; Corso, M.; Vasseur, G.; Rogero, C.; Guitian, E.; Pascual, J. I.; Ortega, J. E.; Wakayama, Y.; Pena, D. ACS Nano 2016, 10, 9000.

(8) Talirz, L.; Ruffieux, P.; Fasel, R. Avd. Mater. 2016, 28, 6222 .

(9) Sakaguchi, H.; Song, S.; Kojima, T.; Nakae, T. Nat. Chem. 2017, 9, 57.

(10) Kawai, S.; Saito, S.; Osumi, S.; Yamaguchi, S.; Foster, A. S.; Spijker, P.; Meyer, E. Nat. Commun. 2015, 6, 8098.

(11) Ye, R.; Xiang, C.; Lin, J.; Peng, Z.; Huang, K.; Yan, Z.; Cook, N. P.; Samuel, E. L. G.; Hwang, C.-C.; Ruan, G.; Ceriotti, G.; Raji, A.-R. O.; Martí, A. A.; Tour, J. M. Nature Communications 2013, 4, 2943.

(12) Chen, Z.; Lin, Y.-M.; Rooks, M. J.; Avouris, P. Physica E: Low-dimensional Systems and Nanostructures 2007, 40, 228.

(13) Kosynkin, D. V.; Higginbotham, A. L.; Sinitskii, A.; Lomeda, J. R.; Dimiev, A.; Price, B. K.; Tour, J. M. Nature 2009, 458, 872.

(14) Narita, A.; Feng, X.; Hernandez, Y.; Jensen, S. A.; Bonn, M.; Yang, H.; Verzhbitskiy, I. A.; Casiraghi, C.; Hansen, M. R.; Koch, A. H. R.; Fytas, G.; Ivasenko, O.; Li, B.; Mali, K. S.; Balandina, T.; Mahesh, S.; De Feyter, S.; Müllen, K. Nat. Chem. 2014, 6, 126.

(15) Narita, A.; Verzhbitskiy, I. A.; Frederickx, W.; Mali, K. S.; Jensen, S. A.; Hansen, M. R.; Bonn, M.; De Feyter, S.; Casiraghi, C.; Feng, X.; Müllen, K. ACS Nano 2014, $8,11622$.

(16) Zhao, S.; Rondin, L.; Delport, G.; Voisin, C.; Beser, U.; Hu, Y.; Feng, X.; Müllen, K.; Narita, A.; Campidelli, S.; Lauret, J. S. Carbon 2017, 119, 235.

(17) Ivanov, I.; Hu, Y.; Osella, S.; Beser, U.; Wang, H. I.; Beljonne, D.; Narita, A.; Müllen, K.; Turchinovich, D.; Bonn, M. J. Am. Chem. Soc. 2017, 139, 7982.

(18) Soavi, G.; Dal Conte, S.; Manzoni, C.; Viola, D.; Narita, A.; Hu, Y.; Feng, X.; Hohenester, U.; Molinari, E.; Prezzi, D.; Müllen, K.; Cerullo, G. Nat. Commun. 2016, 7, 11010 .

(19) Wu, Z. S.; Parvez, K.; Feng, X.; Müllen, K. Nat. Commun. 2013, 4.

(20) Baumgartner, R.; Fu, H.; Song, Z.; Lin, Y.; Cheng, J. Nat. Chem. 2017, 9, 614.
(21) Tan, Y.-Z.; Yang, B.; Parvez, K.; Narita, A.; Osella, S.; Beljonne, D.; Feng, X.; Müllen, K. Nat. Commun. 2013, 4.

(22) Rogers, C.; Perkins, W. S.; Veber, G.; Williams, T. E.; Cloke, R. R.; Fischer, F. R. J. Am. Chem. Soc. 2017, $139,4052$.

(23) Huang, Y.; Mai, Y.; Beser, U.; Teyssandier, J.; Velpula, G.; van Gorp, H.; Straasø, L. A.; Hansen, M. R.; Rizzo, D.; Casiraghi, C.; Yang, R.; Zhang, G.; Wu, D.; Zhang, F.; Yan, D.; De Feyter, S.; Müllen, K.; Feng, X. J. Am. Chem. Soc. 2016, 138, 10136.

(24) Keerthi, A.; Slota, M.; Myers, W. K.; Tretyakov, E.; Baumgarten, M.; Ardavan, A.; Sadeghi, H.; Lambert, C. J.; Narita, A.; Bogani, L.; Müllen, K. Submitted 2017.

(25) Keerthi, A.; Hou, I. C.; Marszalek, T.; Pisula, W.; Baumgarten, M.; Narita, A. Chem Asian J 2016, 11, 2710.

(26) Yukinori, N.; Takahisa, M. Bull. Chem. Soc. Jpn. 1981, 54, 1575.

(27) Verzhbitskiy, I. A.; Corato, M. D.; Ruini, A.; Molinari, E.; Narita, A.; Hu, Y.; Schwab, M. G.; Bruna, M.; Yoon, D.; Milana, S.; Feng, X.; Müllen, K.; Ferrari, A. C.; Casiraghi, C.; Prezzi, D. Nano Letters 2016, 16, 3442.

(28) Guo, Z.; Zhang, D.; Gong, X.-G. Appl. Phys. Lett. 2009, 95, 163103.

(29) Gillen, R.; Mohr, M.; Maultzsch, J. Phys. Rev. B 2010, 81, 205426. 


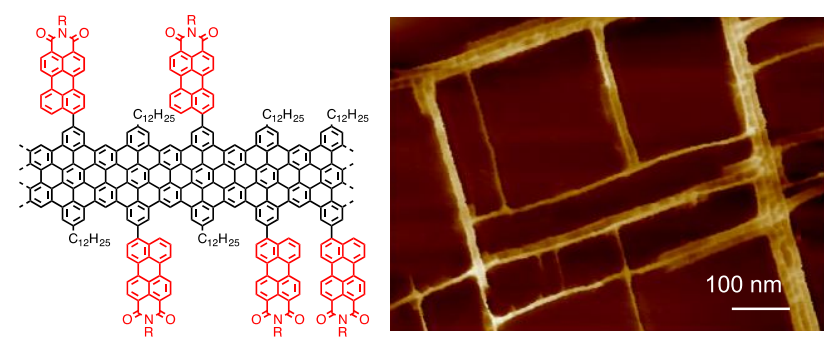